\title{
Interactive comment on "Viscosities, diffusion coefficients, and mixing times of intrinsic fluorescent organic molecules in brown limonene secondary organic aerosol and tests of the Stokes-Einstein equation" by Dagny A. Ullmann et al.
}

\section{Anonymous Referee \#1}

Received and published: 29 September 2018

The authors report about viscosity and diffusivity measurements of a brown carbon containing limonene SOA produced by ozonolysis under high mass loading conditions with subsequent exposure to ammonia. They report an increase in viscosity by 3-5 orders upon changing the water activity from 0.9 to dry and use the measured diffusion coefficient to deduce the mixing times for atmospheric particles. Their result suggest that mixing times are below 1 hour for PBL-conditions. This is in contrast to previous studies looking at SOA under low mass loading conditions which report significantly

Printer-friendly version

Discussion paper 
longer mixing times. The authors compare their viscosity data with their diffusivity data using the Stokes-Einstein relationship and conclude that it holds accurately for viscosities up to $10^{\wedge} 4 \mathrm{~Pa}$ s.

This is a well-written manuscript and its topic is of core interest to the readers of ACP. It makes a very elegant use of the FRAP technique as it uses the $\mathrm{BrC}$ contained in the SOA as the fluorophore. However, the results suffer from being obtained under high mass loading conditions, which make them less relevant for direct applications to atmospheric SOA. As such the calculated mixing times likely provide a lower limit for atmospheric limonene SOA particles. I recommend to the authors to add a discussion whether it would be conceivable to use their technique also for brown LSOA produced at lower mass loading, so that the reader get a better feeling for the limits of the technique.

I recommend publishing the paper as is.

Minor comments:

- Abstract: While the authors acknowledge the problem of high mass loading here, I feel they should state explicitly that the magnitude of the difference in mixing times to more realistic SOA may be 3 orders of magnitude.

- Line 211: I am not entirely convinced by this argument. I agree that thermal steady state will be reached much quicker than the characteristic diffusion time. However, equally important may be the steady-state temperature difference. If significant, such a temperature difference may cause a redistribution of water molecules to outside of the irradiated region changing water activity locally. From the power density and thermal conductivity in the experiments the authors may estimate the temperature increase of the illuminated region.

- Line 253: I assume in Fig. 4, these are all above continents, correct?

Printer-friendly version

- Caption of Fig. 5: Does the uncertainty for the calculated diffusivity include the un- 
certainty of the fluorophore radius?

Interactive comment on Atmos. Chem. Phys. Discuss., https://doi.org/10.5194/acp-2018-899, 2018.

Interactive

comment 